KUNS-1888

hep-th/0312298

December 2003

\title{
A mechanism of the large-scale damping in the CMB anisotropy
}

\author{
Masafumi Fukuma*, Yuji Kono ${ }^{\dagger}$ and Akitsugu Miwa ${ }^{\ddagger}$ \\ Department of Physics, Kyoto University, Kyoto 606-8502, Japan
}

\begin{abstract}
We present a mechanism through which a certain class of short-distance cutoff affects the CMB anisotropies at large angular scales. Our analysis is performed in two steps. The first is given in an intuitive way, using the property of the inflationary universe that quantum fluctuations of an inflaton field become classical after crossing the Hubble horizon. We give a condition for a cutoff to yield a damping on large scales, and show that the holographic cutoff introduced in the preceding paper (hep-th/0307029) does satisfy the condition. The second analysis is carried out by setting an initial condition such that each mode of inflaton starts as the vacuum fluctuation of the Hamiltonian when being released from the constraint of cutoff. The first intuitive discussion is then shown to be correct qualitatively.
\end{abstract}

\footnotetext{
${ }^{*}$ E-mail: fukuma@gauge.scphys.kyoto-u.ac.jp

${ }^{\dagger}$ E-mail: kono@gauge.scphys.kyoto-u.ac.jp

${ }^{\ddagger}$ E-mail: akitsugu@gauge.scphys.kyoto-u.ac.jp
} 


\section{Introduction}

Great progress has recently been made in observational cosmology. In particular, the elaborate measurement of the CMB anisotropy [1,2,3,4] has shown that it can be well explained by the inflationary models [5, 6, 7, ,8, and has determined many of the relevant cosmological parameters from the angular power spectrum $C_{l}$, which is defined through the two-point correlators of the temperature fluctuations as

$$
\left\langle\frac{\delta T}{T}\left(\Omega_{1}\right) \frac{\delta T}{T}\left(\Omega_{2}\right)\right\rangle=\sum_{l \geq 1} \frac{2 l+1}{4 \pi} C_{l} P_{l}\left(\cos \theta_{12}\right) .
$$

Here the bracket \langle\rangle is the sample average taken from various pairs of directions $\Omega_{1}$ and $\Omega_{2}$ on the celestial sphere with the fixed angle $\theta_{12}$.

Some discrepancies are known to exist between the observed data and the theoretical prediction [3]. The main contributions to the excess $\chi^{2}$ originate from scales around the first acoustic peak. Besides them the observed anisotropies have much smaller values at large angular scales. In fact, as for the latter discrepancy, the observational data show that $C_{l}$ is almost proportional to $1 / l(l+1)$ for small $l(10 \lesssim l<50)$, consistent with the almost scale invariant power spectrum predicted by the inflationary models. However, for much smaller $l(l<10)$, the data show that $C_{l}$ 's are much less than the predicted values [3, 9]. The main purpose of the present paper is to discuss this "large-scale damping" as a remnant of the Planck-scale physics.

The conventional explanation of this large-scale damping is based on the so-called cosmic variance (see, e.g., [10,11,12]). In fact, for small $l$, one can take only a few independent

samples $(\sim 2 l+1)$, and thus the observed values can deviate largely from the theoretical mean values. However, if this deviation is not simply a statistical deviation, then it has a possibility to be a clue to some unknown dynamics at the early stage during inflation. In fact, as will be reviewed in the next section, quantum fluctuations of an inflaton field become classical after crossing the Hubble horizon, and the CMB anisotropies at large angular scales are directly related to those classical fluctuations of the inflaton field on large scales. Since the larger-scale modes cross the Hubble horizon and become classical at earlier times, the CMB anisotropies at large angular scales are more sensitive to dynamics at the early stage during inflation.

The idea to modify the power spectrum on large scales has a long history (see, e.g., Refs. [13, 14, 15]). In particular, understanding of trans-Planckian physics has been recognized as an important subject. The effects of trans-Planckian physics on the CMB anisotropies are 
discussed in various aspects in Refs. 16, 17, 18, 19, 20, 21, 22, 23, 24, 25,.

The most promising candidate that can explain the dynamics of quantum gravity at the Planck scale is string theory. One of the basic results in string theory is the existence of the minimum length scale $l_{s}$ [26, 27], and spacetime is expected to loose its smooth Riemannian structure and to become discrete (or noncommutative) at the Planck scale. At the present moment, however, we do not have an analytic tool in hand with which string dynamics around the Planck scale can be dealt with in a definite manner. In the present paper, we assume that such quantum effects of gravity can be reflected simply by introducing a cutoff or a noncommutative scale $L_{\text {cut }}\left(=O\left(l_{s}\right)\right)$ into the dynamics of the inflaton field. We try to understand the large-scale damping by introducing a holographic cutoff to an inflaton field around the Planck scale.

The possibility that the large-scale damping could be explained by a noncommutative nature of spacetime was first investigated in Ref. [28. ${ }^{1}$ The main aim of the present article is to complement the discussions given there, and also to clarify what kind of cutoff should be chosen in order to have the large-scale damping in the CMB anisotropy.

This paper is organized as follows. In section 2, we explain a mechanism of the largescale damping, using the property of the inflationary universe that quantum fluctuations of an inflaton field become classical after crossing the Hubble horizon, and we show that our previous result given in Ref. [28] can be naturally understood along this argument. However, this process of "classicalization" should be incorporated automatically once a proper initial condition is set when an inflaton field starts its quantum fluctuations. In section 3, we discuss what initial condition should be set in the presence of the cutoff introduced in Ref. 28 and analyze the power spectrum of the inflaton field at the exit time of inflation. Section 4 is devoted to conclusion and outlook.

\section{A mechanism of the large-scale damping}

We start our discussion with recalling that the currently observed CMB power spectrum on the superhorizon scale is proportional to the power spectrum of the inflaton field at the exit time of inflation. We then introduce cutoff in various ways, and investigate which kind of cutoff will give rise to the large-scale damping.

\footnotetext{
${ }^{1}$ See Refs. [18,19,20,21,22, for inflationary models using noncommutative geometry in different contexts.
} 


\subsection{Classicalization process and the CMB power spectrum in in- flationary models (review)}

The flat FRW metric during inflation is given by

$$
\begin{aligned}
& d s^{2}=g_{\mu \nu} d x^{\mu} d x^{\nu}=a^{2}(\eta)\left(-d \eta^{2}+d \boldsymbol{x}^{2}\right)=a^{2}(\eta)\left(-d \eta^{2}+d r^{2}+r^{2} d \Omega^{2}\right), \\
& a(\eta)=-\frac{1}{H \eta} \quad(\eta<0) .
\end{aligned}
$$

Here $a(\eta)$ is the scale factor and $\eta$ is the conformal time. Note that $\eta$ is negative during inflation and the exit time of inflation is given by $\eta \rightarrow-0$. The inflaton field $\Phi(\eta, \boldsymbol{x})$ is decomposed into the classical part $\bar{\phi}(\eta)$ and the fluctuation $\phi(\eta, \boldsymbol{x})$ around it. We only consider the Gaussian fluctuation, which is realized by taking the part quadratic in $\phi$ after substituting $\Phi=\bar{\phi}+\phi$ into the action $S[\Phi]=\int d^{4} x \sqrt{-g}\left(-(1 / 2) g^{\mu \nu} \partial_{\mu} \Phi \partial_{\nu} \Phi-V(\Phi)\right)$. We also assume that the potential $V(\Phi)$ has a plateau which is flat enough to ensure that the classical solution $\bar{\phi}$ can roll very slowly. Thus, in the zeroth order of the slow roll approximation we can simply neglect the potential term and obtain

$$
\begin{aligned}
S & =S_{\mathrm{cl}}+S_{\text {fluct }}, \\
S_{\text {fluct }} & =-\frac{1}{2} \int d^{4} x \sqrt{-g} g^{\mu \nu} \partial_{\mu} \phi \partial_{\nu} \phi .
\end{aligned}
$$

$S_{\text {fluct }}$ represents a free scalar field in de Sitter spacetime.

In order to quantize a free scalar field on a curved spacetime, one begins with considering a complete set of normalized, positive-energy solutions to the Klein-Gordon equation [29],

$$
\nabla^{2} \psi_{A}=0
$$

Here $A$ labels the modes with respect to the comoving coordinates, and the functions $\psi_{A}$ are normalized as

$$
\begin{array}{ll}
\left(\psi_{A}, \psi_{B}\right)=\delta_{A B}, & \left(\psi_{A}, \psi_{B}^{*}\right)=0, \\
\left(\psi_{A}^{*}, \psi_{B}\right)=0, & \left(\psi_{A}^{*}, \psi_{B}^{*}\right)=-\delta_{A B},
\end{array}
$$

with respect to the symplectic product $($,$) that is defined for arbitrary complex functions$ $f$ and $g$ as

$$
(f, g) \equiv i \int_{\Sigma} d S^{\mu}\left(f^{*} \partial_{\mu} g-\partial_{\mu} f^{*} g\right)=(g, f)^{*}=-\left(f^{*}, g^{*}\right)^{*}
$$


where $\Sigma$ is a given spacelike hypersurface, and $d S^{\mu}$ is its three-dimensional volume element. Note that $(f, g)$ is independent of the choice of $\Sigma$ when $f$ and $g$ satisfy the Klein-Gordon equation. Then by introducing the corresponding annihilation and creation operators $\left\{a_{A}\right\}$ and $\left\{a_{A}^{\dagger}\right\}$ with the commutation relations

$$
\left[a_{A}, a_{B}^{\dagger}\right]=\delta_{A B}
$$

the quantum field $\phi$ is represented as

$$
\phi=\sum_{A}\left(a_{A} \psi_{A}+a_{A}^{\dagger} \psi_{A}^{*}\right)
$$

For later convenience, we denote by $k_{A}$ the magnitude of the comoving wave number of the mode $A$.

The set of modes $\{A\}$ can be chosen as one likes, depending on which symmetry is kept manifest. We here give a few examples:

$\underline{\text { (1) } A=\boldsymbol{k}}$ : Three-dimensional translational symmetry is kept manifest, and

$$
\begin{aligned}
\psi_{\boldsymbol{k}}(\eta, \boldsymbol{x}) & =\frac{H}{\sqrt{2 k^{3}}}(1+i k \eta) e^{-i k \eta+i \boldsymbol{k} \cdot \boldsymbol{x}} \quad(k \equiv|\boldsymbol{k}|), \\
\delta_{A A^{\prime}} & =(2 \pi)^{3} \delta^{3}\left(\boldsymbol{k}-\boldsymbol{k}^{\prime}\right), \quad \sum_{A}=\int \frac{d^{3} \boldsymbol{k}}{(2 \pi)^{3}}, \\
k_{A} & =k .
\end{aligned}
$$

(2) $A=(k, l, m):$ Rotational symmetry is kept manifest, and

$$
\begin{aligned}
\psi_{k l m}(\eta, r, \Omega) & =H \sqrt{\frac{2}{k}}(1+i k \eta) e^{-i k \eta} j_{l}(k r) Y_{l m}(\Omega) \equiv \psi_{k l}(\eta, r) Y_{l m}(\Omega), \\
\delta_{A A^{\prime}} & =2 \pi \delta\left(k-k^{\prime}\right) \delta_{l l^{\prime}} \delta_{m m^{\prime}}, \quad \sum_{A}=\int_{0}^{\infty} \frac{d k}{2 \pi} \sum_{l} \sum_{m} \\
k_{A} & =k
\end{aligned}
$$

where $r=|\boldsymbol{x}|$ and $\Omega=(\theta, \varphi)$ is the angular direction of the comoving coordinate $\boldsymbol{x}^{2}$

\footnotetext{
${ }^{2}$ In the above, both of the positive-energy solutions are chosen such that they behave as $e^{-i k \eta}$ in the limit $\eta \rightarrow-\infty$. It is easy to see that the annihilation operators and the positive-energy solutions for these two sets of modes are related as

$$
\begin{aligned}
& a_{k l m}=\frac{i^{l} k}{2 \pi} \int d^{2} \Omega_{\boldsymbol{k}} Y_{l m}^{*}\left(\Omega_{\boldsymbol{k}}\right) a_{\boldsymbol{k}} \\
& \psi_{\boldsymbol{k}}(\eta, \boldsymbol{x})=\frac{2 \pi}{k} \sum_{l, m} i^{l} Y_{l m}^{*}\left(\Omega_{\boldsymbol{k}}\right) \psi_{k l m}(\eta, r, \Omega),
\end{aligned}
$$

where $\Omega_{\boldsymbol{k}}$ is the angular direction of the comoving wave number $\boldsymbol{k}$.
} 
When rotational invariance is manifest, it is often convenient to decompose the field as

$$
\phi(\eta, r, \Omega)=\sum_{l, m} \phi_{l m}(\eta, r) Y_{l m}(\Omega) \quad\left(\text { or } \quad \phi_{l m}(\eta, r)=\int d^{2} \Omega Y_{l m}^{*}(\Omega) \phi(\eta, r, \Omega)\right),
$$

and treat the coefficient

$$
\phi_{l m}(\eta, r)=\int_{0}^{\infty} \frac{d k}{2 \pi}\left[\psi_{k l}(\eta, r) a_{k l m}+(-1)^{m} \psi_{k l}^{*}(\eta, r) a_{k l-m}^{\dagger}\right]
$$

as a field over a two-dimensional spacetime $(\eta, r)$. The angular power spectrum of the inflaton field, $C_{l}^{\phi}$, is then defined by

$$
C_{l}^{\phi}(\eta, r) \equiv\left\langle 0\left|\phi_{l m}^{\dagger}(\eta, r) \phi_{l m}(\eta, r)\right| 0\right\rangle=\int_{0}^{\infty} \frac{d k}{2 \pi}\left|\psi_{k l}(\eta, r)\right|^{2}
$$

Cosmological perturbation theory [33, 12] predicts that at large angular scales (i.e., for small l's) the angular power spectrum $C_{l}$ of the CMB anisotropy observed at present is proportional to that of the inflaton at the exit time of inflation,

$$
C_{l} \propto \lim _{\eta \rightarrow-0} C_{l}^{\phi}\left(\eta, r_{*}\right)
$$

Here $r=r_{*}$ is the comoving radius of the last scattering surface (LSS), which is a two-sphere

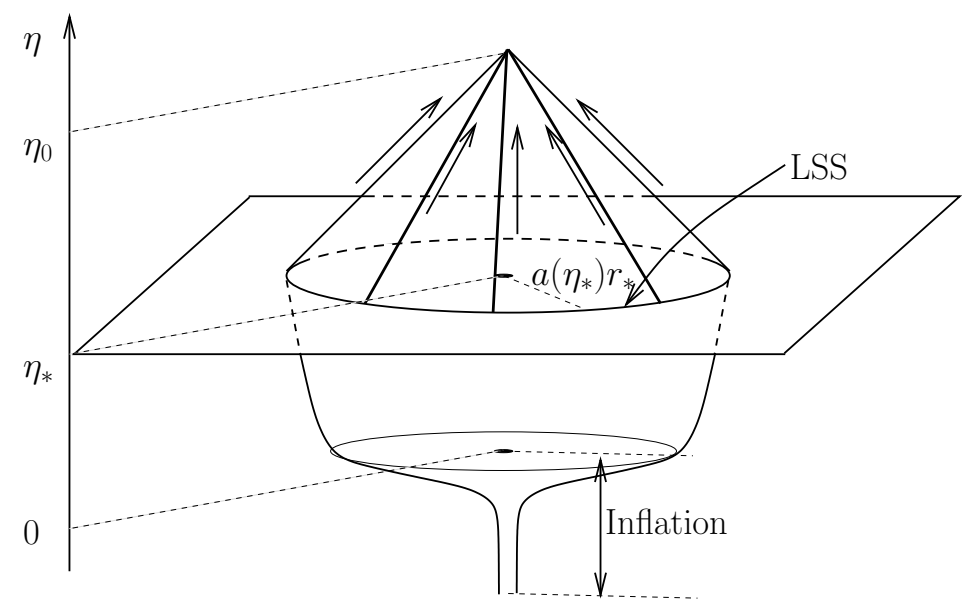

Figure 1: An observer at the present time $\eta=\eta_{0}$ sees photons from the LSS as the CMB. By setting the comoving radius of the LSS to be $r=r_{*}$, the LSS is traced back to a two-sphere with small physical (proper) radius $r_{\text {phys }}=a(\eta) r$ during inflation. The moment $\eta=0$ is the exit time of inflation.

on the time slice (at $\eta=\eta_{*}$ ) when the recombination takes place (see Fig. 10). The relation (2.21) can be understood as follows.

First, in the zeroth order of the slow roll approximation the Hubble length $1 / H$ can be thought to be constant and the spacetime becomes de Sitter. In this approximation, once 
the inflation starts, two points separated with a distance longer than the Hubble length cannot exchange information until the end of the inflation. ${ }^{3}$ Thus, a mode $A$ can fluctuate quantum mechanically only when its physical wave length $\lambda_{A}$ is shorter than the Hubble length. However, in the expanding universe, the physical wave length of the mode $A$ is a monotonically increasing function of time as $\lambda_{A}(\eta)=a(\eta) / k_{A}$, while the Hubble length is constant in time during inflation. Thus, as the physical wave length increases, the mode $A$ gradually looses its quantum nature and eventually becomes purely classical at the exit time of inflation $\eta \rightarrow-0$ (for a detailed analysis, see, e.g., Ref. [31]). This process is sometimes called the classicalization. The characteristic moment $\eta_{A}^{\mathrm{C}}$ at which the crossover from quantum to classical physics occurs for the mode $A$, can be evaluated by setting $\lambda_{A}=1 / H$ (crossing the Hubble horizon) and is found to be $\eta_{A}^{\mathrm{C}}=-1 / k_{A}$ (see Fig. 2). We thus can schematically say that

$$
\begin{aligned}
& \eta<\eta_{A}^{\mathrm{C}} \longrightarrow \text { the mode } A \text { can fluctuate quantum mechanically, } \\
& \eta>\eta_{A}^{\mathrm{C}} \longrightarrow \text { the mode } A \text { cannot fluctuate quantum mechanically. }
\end{aligned}
$$

Note that a mode of smaller $k_{A}$ crosses the horizon at an earlier time.

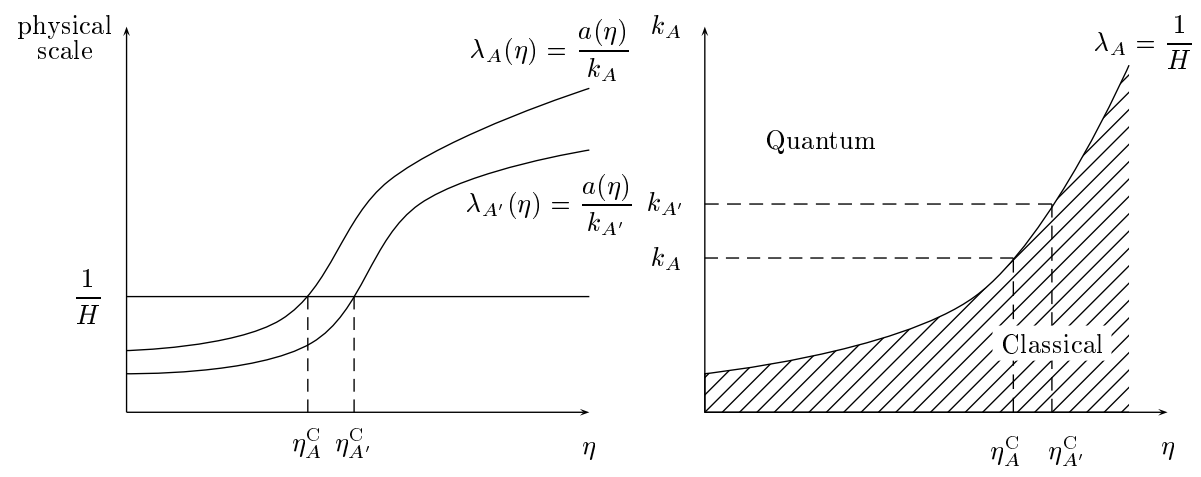

Figure 2: The curve of $\eta_{A}^{\mathrm{C}}$ at which the mode $A$ crosses the horizon and becomes classical.

After the ending of inflation, one can apply the linear perturbation theory, regarding $\phi_{l m}(\eta, r)$ in Eq. (2.20) simply as a classical perturbation. One can show that the CMB anisotropies on large scales are proportional to the perturbation of gravitational potential on the LSS (Sachs-Wolfe effect [32,12]). The latter in turn can be linearly related to the classical fluctuations of the inflaton at the exit time of inflation by using the conservation law in the cosmological perturbation theory which holds on the superhorizon scale with

\footnotetext{
${ }^{3}$ The Hubble length actually comes to depend on time and the spacetime deviates from de Sitter, if corrections beyond the zeroth order slow roll approximation are taken into account. In this case, one should be careful in analyzing the correlation between two points in terms of the Hubble length. See Ref. 30.
} 
adiabatic perturbation [33, 12]. Putting it all together, the angular mode $c_{l m}$ of the CMB anisotropy

$$
\frac{\delta T}{T}(\Omega)=\sum_{l \geq 1} \sum_{m=-l}^{l} c_{l m} Y_{l m}(\Omega)
$$

is proportional to $\phi_{l m}\left(\eta=-0, r=r_{*}\right)$, and thus, the angular power spectrum $C_{l}$ of the CMB anisotropy observed at the present time is proportional to that of the inflaton field at the exit time of inflation:

$$
C_{l}=\left\langle\left|c_{l m}\right|^{2}\right\rangle \propto \lim _{\eta \rightarrow-0}\left\langle\left|\phi_{l m}\left(\eta, r_{*}\right)\right|^{2}\right\rangle=\lim _{\eta \rightarrow-0} C_{l}^{\phi}\left(\eta, r_{*}\right)
$$

In the zeroth order of the slow roll approximation, we have $\lim _{\eta \rightarrow-0} \psi_{k l}\left(\eta, r_{*}\right)=H \sqrt{2 / k} j_{l}\left(k r_{*}\right)$, so that

$$
C_{l} \propto C_{l}^{\phi}\left(0, r_{*}\right)=\frac{H^{2}}{\pi} \int_{0}^{\infty} \frac{d k}{k}\left(j_{l}\left(k r_{*}\right)\right)^{2} \propto \frac{1}{l(l+1)}
$$

This prediction from inflationary models agrees quite well with the observational data at large angular scales.

\subsection{Time-dependent cutoff and a criterion for the large-scale damp- ing}

In this subsection, we discuss how the previous analysis should be modified when various types of cutoff are introduced. One may think that nothing would change on large scales since the short-distance cutoff usually does not play important roles on large scales in local quantum field theories. However, in the expanding universe, the cutoff can be a timedependent function for the comoving modes. This fact, combined with the classicalization process, proves to give rise to large suppression on the large-scale modes when a particular form of cutoff is chosen.

Suppose that we introduce a short-distance cutoff $L_{\text {cut }}$ on the physical distance scale such that it is constant in time. Then there appears another moment which will be important in the following discussion. In fact, as we see below, this leads to a cutoff $\Lambda_{\text {cut }}(\eta)$ on comoving modes, which is generically a monotonically increasing function of time in the expanding universe (see Fig. 3), and whose dependence on the short-distance cutoff $L_{\text {cut }}$ strongly depends on the way of introducing $L_{\text {cut }}$ into a system. A comoving mode $A$ can

exist as a quantum fluctuation only when the inequality $k_{A} \leq \Lambda_{\text {cut }}(\eta)$ is satisfied. We thus 


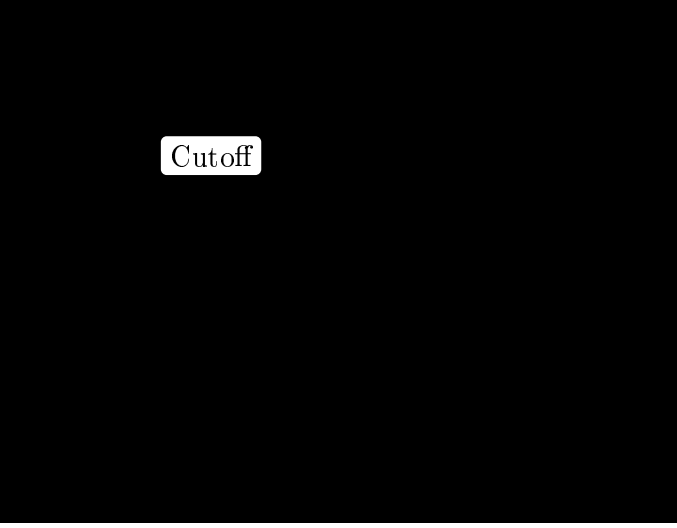

Figure 3: The time-dependent comoving cutoff. In the shadowed region, quantum fluctuations are prohibited. The mode $A$ starts its quantum fluctuation at the moment $\eta=\eta_{A}^{\mathrm{Q}}$.

can introduce the moment $\eta_{A}^{\mathrm{Q}}$ at which the mode $A$ starts its quantum fluctuation upon being released from the constraint of the cutoff. In a nutshell,

$$
\begin{aligned}
& \eta<\eta_{A}^{\mathrm{Q}} \longrightarrow \text { the mode } A \text { is prohibited to exist as a quantum fluctuation, } \\
& \eta>\eta_{A}^{\mathrm{Q}} \longrightarrow \text { the mode } A \text { is allowed to fluctuate quantum mechanically. }
\end{aligned}
$$

In order to calculate the power spectrum we need to fix the initial condition at $\eta=\eta_{A}^{\mathrm{Q}}$ for each mode $A$. Although we have no established one, we know which form the initial conditions should take for the modes in the two extreme cases, $\eta_{A}^{\mathrm{Q}} \ll \eta_{A}^{\mathrm{C}}$ and $\eta_{A}^{\mathrm{C}} \ll \eta_{A}^{\mathrm{Q}}$. In the former case, the physical length scales of such modes $A$ upon being created are much smaller than the Hubble length. Therefore, as is usual, the Bunch-Davies vacuum [29] is favored and the contribution of these modes to the CMB anisotropy should be the same as Eq. (2.24). On the other hand, as for the latter case $\eta_{A}^{\mathrm{C}} \ll \eta_{A}^{\mathrm{Q}}$, we first recall that the anisotropies of the CMB observed at the present time correspond to the classical values (or the 'fossils') of the quantum fluctuations of the inflaton field (see the discussion in the previous subsection). We also note that a mode $A$ with $\eta_{A}^{\mathrm{C}} \ll \eta_{A}^{\mathrm{Q}}$ must be already classical well before its quantum fluctuation starts. Since no fossil can exist if the mode does not have a life of quantum fluctuation, such a mode with $\eta_{A}^{\mathrm{C}} \ll \eta_{A}^{\mathrm{Q}}$ has no classical amplitude and thus does not contribute to the CMB anisotropy.

In between these two limits, we do not a priori know how modes contribute to the CMB anisotropies without setting definite initial conditions on quantum fluctuations. We consider this problem in detail in section 3. Here, we instead set the following ansatz, expecting from the above argument that those modes with $\eta_{A}^{\mathrm{Q}}<\eta_{A}^{\mathrm{C}}$ contribute to the CMB anisotropies more than those with $\eta_{A}^{\mathrm{C}}<\eta_{A}^{\mathrm{Q}}$ do: 


\section{$\underline{\text { Ansatz }}$}

$$
\begin{cases}\bullet \eta_{A}^{\mathrm{C}}<\eta_{A}^{\mathrm{Q}} \longrightarrow & \text { the mode } A \text { does not contribute to the CMB anisotropy, } \\ \bullet \eta_{A}^{\mathrm{Q}}<\eta_{A}^{\mathrm{C}} \longrightarrow & \text { the mode } A \text { contributes to the CMB anisotropy as in } \\ & \text { the absence of cutoff. }\end{cases}
$$

Whether the cutoff gives rise to a damping of the CMB anisotropy at large distance scales or at short distance scales, will depend on the way of introducing cutoff. If $\eta_{A}^{\mathrm{Q}}$ and

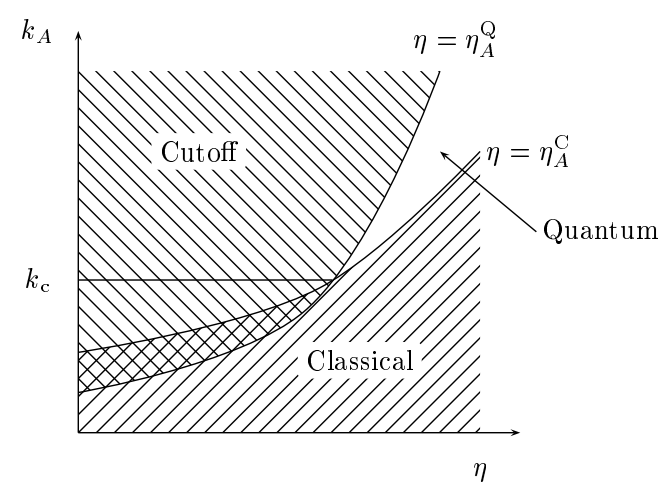

Figure 4: Case in which a damping occurs on scales larger than $1 / k_{\mathrm{c}}$.

$\eta_{A}^{\mathrm{C}}$ behave as in Fig. 4, then larger-scale modes do not contribute and the CMB anisotropy has a damping on large scales. On the other hand, if their behaviors are as in Fig. 5 , then

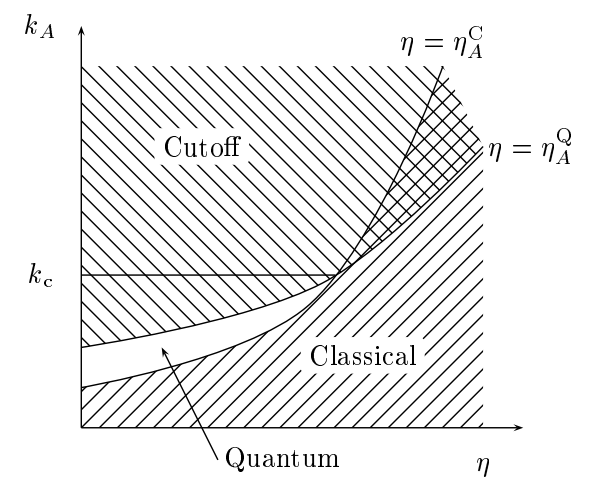

Figure 5: Case in which a damping occurs on scales smaller than $1 / k_{\mathrm{c}}$.

one would observe a damping on small scales. In the case of Fig. 6, there are no quantum modes and will be no classical fluctuations, while in the case of Fig. [7 all the quantum modes are allowed to exist, and one would not see a major deviation from the case in the absence of cutoff. 


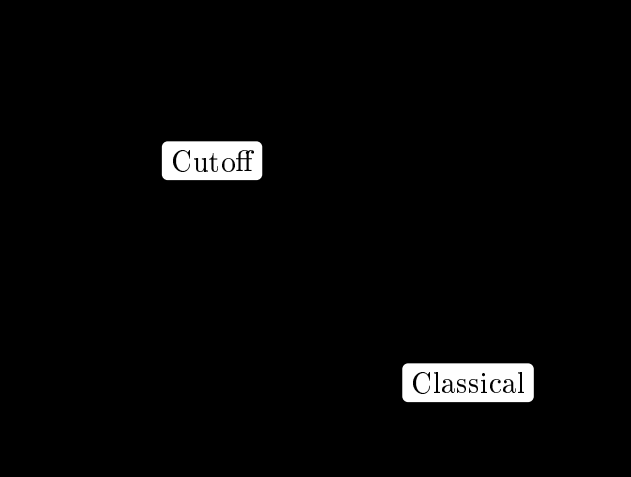

$\eta$

Figure 6: Case in which no quantum fluctuations occur during the whole period. No CMB anisotropies will be made.

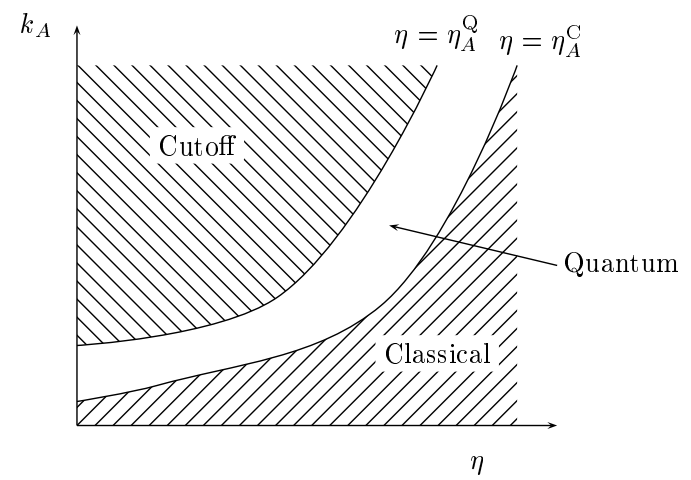

Figure 7: Case in which all the quantum fluctuations are allowed to exist during inflation. This will give almost the same result with that in the absence of cutoff. 


\subsection{A few examples}

We give a few examples on the way of introducing cutoff. In the following, we expand the inflaton field $\phi(\eta, r, \Omega)$ with respect to angular coordinates as

$$
\phi(\eta, r, \Omega)=\sum_{l \geq 0} \sum_{m=-l}^{l} \int_{0}^{\infty} \frac{d k}{2 \pi} \phi_{k l m}(\eta) j_{l}(k r) Y_{l m}(\Omega),
$$

and label the mode as $A=(k, l, m)$. In Eq. (2.26),$k$ denotes the comoving wave number in the radial direction and $(l, m)$ represents the multipole in the angular directions.

\subsubsection{Homogeneous cutoff on the physical wave length}

We first consider the case in which a time-independent short-distance cutoff $L_{\text {cut }}$ is put on the physical wave length. That is, we require that the mode $A$ can exist only when the following inequality holds:

$$
\frac{a(\eta)}{k} \geq L_{\mathrm{cut}}
$$

This can be imagined as that the three-dimensional space is a lattice with the spacing $L_{\text {cut }}$.

By setting

$$
\frac{a\left(\eta_{A}^{\mathrm{Q}}\right)}{k}=-\frac{1}{k H \eta_{A}^{\mathrm{Q}}} \equiv L_{\mathrm{cut}},
$$

the moment $\eta_{A}^{Q}$ at which the mode starts its quantum fluctuation is found to be

$$
\eta_{A}^{\mathrm{Q}}=-\frac{1}{k L_{\mathrm{cut}} H}
$$

On the other hand, the moment $\eta_{A}^{\mathrm{C}}$ at which the mode crosses the horizon and becomes classical, is determined by setting

$$
\lambda_{A}=\frac{a\left(\eta_{A}^{\mathrm{C}}\right)}{k}=-\frac{1}{k H \eta_{A}^{\mathrm{C}}} \equiv \frac{1}{H},
$$

or

$$
\eta_{A}^{\mathrm{C}}=-\frac{1}{k}
$$

$\eta_{A}^{\mathrm{C}}$ and $\eta_{A}^{\mathrm{Q}}$ are depicted in Fig. 8, where the vertical axis represents $k_{A}=k$. This figure corresponds to Fig. [ or Fig. 7 depending on the value of $L_{\text {cut }} H(>1$ or $<1)$, and thus we conclude that this way of introducing cutoff does not yield a particular damping in the CMB anisotropy. 


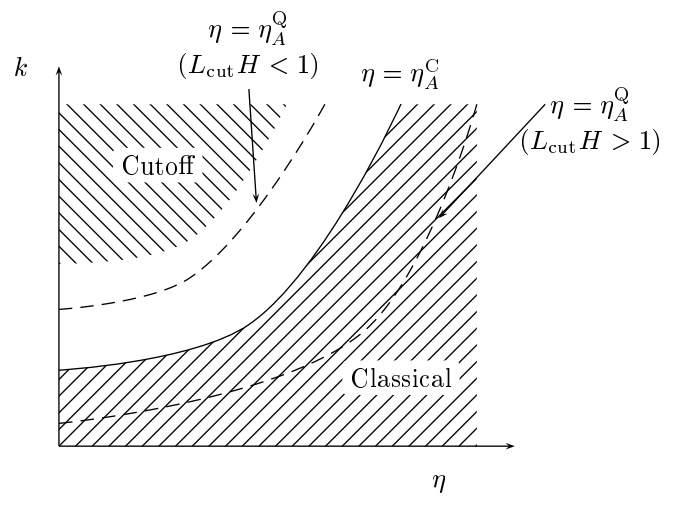

Figure 8: Homogeneous cutoff on the physical wave length. The figure has the same pattern with that of Fig. [ or Fig. 7 depending on the value of $L_{\text {cut }} H$.

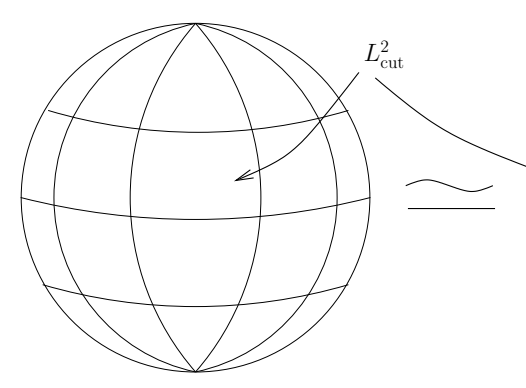

(a)

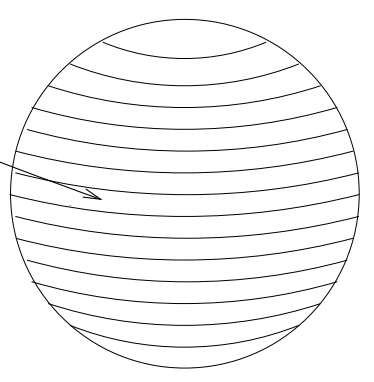

(b)

Figure 9: Fuzzy sphere used as a cutoff. (a) The fuzzy sphere consists of $(N+1)$ points, each of which is represented as a two-dimensional region of area $L_{\text {cut }}^{2}$. (b) By using the noncommutative algebra for the spatial coordinates $\hat{\boldsymbol{x}}=\left(\hat{x}^{1}, \hat{x}^{2}, \hat{x}^{3}\right)$ with the commutation relations $\left[\hat{x}^{i}, \hat{x}^{j}\right]=(2 r i / \sqrt{N(N+2)}) \epsilon^{i j k} \hat{x}^{k}$, the fuzzy sphere of radius $r$ is represented as the set of $(N+1)$ looped strips of equal area $L_{\text {cut }}^{2}$ when a particular direction (say, $\hat{x}^{3}$ ) is diagonalized [34.

\subsubsection{Fuzzy sphere}

We next consider the cutoff [28] with which the two-sphere for each $(\eta, r)$ is replaced by a fuzzy sphere, such that only one-bit degrees of freedom can reside on each area $L_{\text {cut }}^{2}$ 34]. Then the fuzzy sphere consists of finitely many points (see Fig. 9), the number of which is given by

$$
\frac{4 \pi a^{2}(\eta) r^{2}}{L_{\text {cut }}^{2}}=\frac{4 \pi r^{2}}{L_{\text {cut }}^{2} H^{2} \eta^{2}} \equiv N(\eta, r)+1 .
$$

This in turn gives an upper bound on $l$ as (see, e.g., [28])

$$
l \leq N(\eta, r)=\frac{4 \pi r^{2}}{L_{\text {cut }}^{2} H^{2} \eta^{2}}-1 .
$$


If we consider the orbit of the LSS with the fixed comoving radius $r=r_{*}$, the moment $\eta_{A}^{\mathrm{Q}}$ is given by solving the equation

$$
l=N\left(\eta_{A}^{\mathrm{Q}}, r_{*}\right)=\frac{4 \pi r_{*}^{2}}{L_{\text {cut }}^{2} H^{2}\left(\eta_{A}^{\mathrm{Q}}\right)^{2}}-1
$$

and thus found to be

$$
\eta_{A}^{\mathrm{Q}}=-\frac{r_{*}}{L_{\text {cut }} H} \sqrt{\frac{4 \pi}{l+1}} .
$$

The moment $\eta_{A}^{\mathrm{C}}$ is again given by

$$
\eta_{A}^{\mathrm{C}}=-\frac{1}{k}
$$

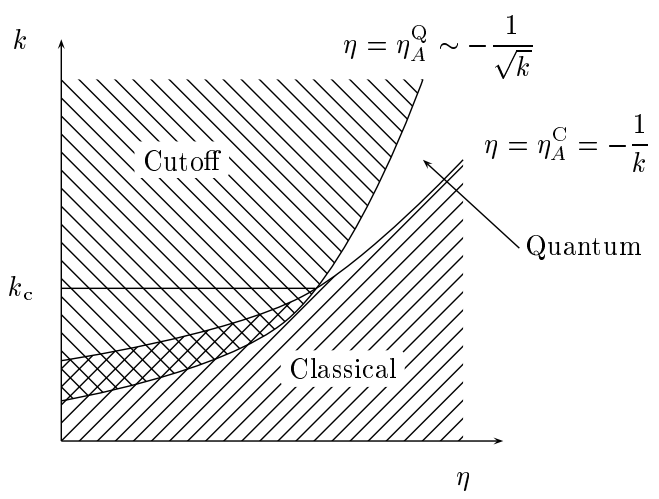

Figure 10: Cutoff with fuzzy sphere. This has the same pattern with that of Fig. 团 and a damping will occur at large distance scales.

These two moments $\eta_{A}^{\mathrm{Q}}$ and $\eta_{A}^{\mathrm{C}}$ can be compared if one notes that $k$ can be identified with $\pi(l+1) / 2 r_{*}$ (i.e., $\left.\eta_{A}^{\mathrm{Q}} \sim-\pi \sqrt{2 r_{*}} /\left(L_{\text {cut }} H \sqrt{k}\right)\right)$ since the spherical Bessel function $j_{l}\left(k r_{*}\right)$ has a sharp peak at the value $k r_{*} \sim \pi(l+1) / 2$. The result is given in Fig. 10. This figure has the same pattern with that of Fig. 4 and thus leads to a damping at large distance scales.

This line of argument was given in Ref. 28]. There the analysis was made by setting the ansatz (2.25) that once a quantum mode has a period of quantum fluctuation, the classical value has the magnitude same with that of the case in the absence of cutoff. This ansatz is equivalent to the prescription that the normalized positive-energy solution in the absence of cutoff,

$$
\psi_{A=(k, l, m)}^{(0)}(\eta, r, \Omega)=H \sqrt{\frac{2}{k}}(1+i k \eta) j_{l}\left(k r_{*}\right) e^{-i k \eta} Y_{l m}(\Omega)
$$


is replaced by

$$
\begin{aligned}
\psi_{A}(\eta, r, \Omega) & \equiv \theta\left(\eta_{A}^{\mathrm{C}}-\eta_{A}^{\mathrm{Q}}\right) \psi_{A}^{(0)}(\eta, r, \Omega) \\
& =\theta\left(k-\frac{\alpha_{l}}{r_{*}}\right) \psi_{A}^{(0)}(\eta, r, \Omega) \quad\left(\alpha_{l} \equiv L_{\mathrm{cut}} H \sqrt{\frac{l+1}{4 \pi}}\right)
\end{aligned}
$$

in the presence of the noncommutativity. This gives an IR cutoff on the integration over the comoving wave number, and the angular power spectrum of inflaton is found to be

$$
\left.\left\langle 0\left|\phi_{l m}^{\dagger}\left(\eta, r_{*}\right) \phi_{l m}\left(\eta, r_{*}\right)\right| 0\right\rangle\right|_{\eta \rightarrow-0}=\frac{H^{2}}{\pi} \int_{\alpha_{l} / r_{*}}^{\infty} \frac{d k}{k}\left(j_{l}\left(k r_{*}\right)\right)^{2} .
$$

If we further introduce the spectral index $n(\sim 1)$, then the angular power spectrum becomes

$$
\left.\left\langle 0\left|\phi_{l m}^{\dagger}\left(\eta, r_{*}\right) \phi_{l m}\left(\eta, r_{*}\right)\right| 0\right\rangle\right|_{\eta \rightarrow-0}=\frac{H^{2}}{\pi} \int_{\alpha_{l} / r_{*}}^{\infty} \frac{d k}{k} k^{n-1}\left(j_{l}\left(k r_{*}\right)\right)^{2}
$$

which gives the angular power spectrum of the CMB anisotropy as

$$
C_{l}=\left(1-\beta_{l}\right) C_{l}^{(0)}
$$

where the $C_{l}^{(0)}$ is the value when noncommutativity is absent during inflation. The damping factor $\left(1-\beta_{l}\right)$ is given by

$$
\beta_{l}=\frac{4}{\sqrt{\pi}} \frac{\Gamma((4-n) / 2) \Gamma(l+(5-n) / 2)}{\Gamma((3-n) / 2) \Gamma(l+(n-1) / 2)} \int_{0}^{\alpha_{l}} d x x^{n-2}\left(j_{l}(x)\right)^{2},
$$

and is depicted in Fig. 11] with $n=0.95$.

\subsection{Connection with the existing literature}

So far we have discussed the effects of cutoff on the CMB anisotropies by comparing the two moments $\eta_{A}^{\mathrm{C}}$ and $\eta_{A}^{\mathrm{Q}}$. In the existing literature, discussions are usually given by introducing an energy scale $M_{c}$, where the modes are supposed to be created (see Refs. 16, 17, 18, 19, 20, 21, 22, 23, 24, 25]). Then the only other scale in the problem is the energy scale of inflation, $H$, and thus the effect of the new scale is expressed by the dimensionless ratio $H / M_{c}$. In the literature it is commonly assumed that $M_{c}$ is almost constant in time. In this subsection we argue that the large-scale damping can occur if $M_{c}$ is a monotonically increasing function of time. We further show that the cutoff based on the fuzzy sphere (subsection 2.3.2) actually leads to $M_{c}$ of this kind.

With the use of $M_{c}$, the moment $\eta_{A}^{\mathrm{Q}}$ at which the quantum mode $A$ is created is character-

ized by the equation $a\left(\eta_{A}^{\mathrm{Q}}\right) / k_{A}=1 / M_{c}\left(\eta_{A}^{\mathrm{Q}}\right)$, where we have assumed the possible dependence 


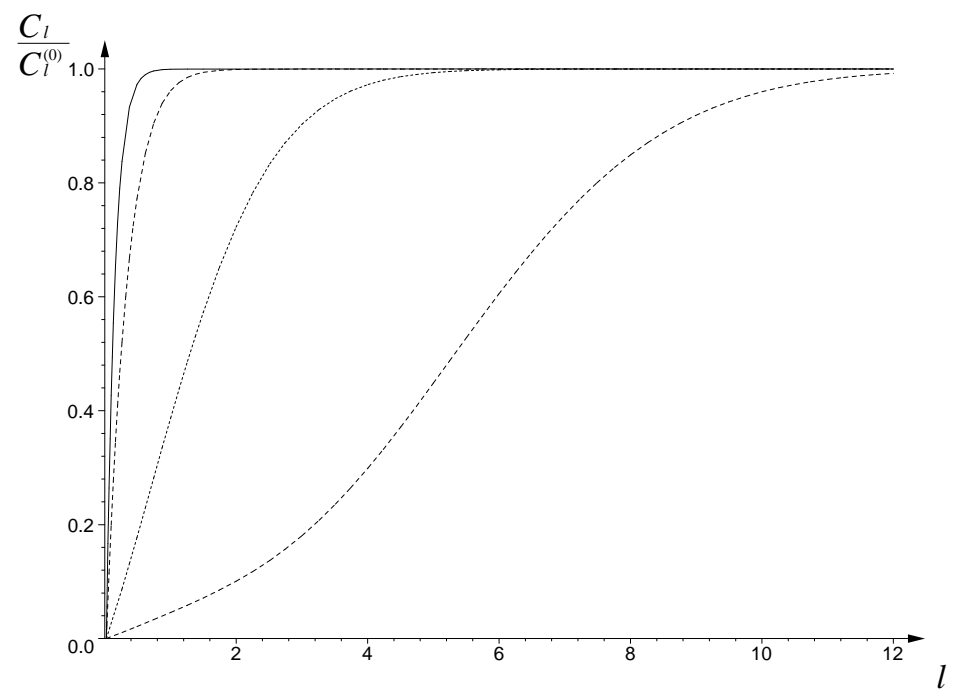

Figure 11: Damping factor for the model using fuzzy sphere. The horizontal axis represents the multipole $l$, while the vertical axis is $C_{l} / C_{l}^{(0)}=1-\beta_{l}$. Here the spectral index is set to be $n=0.95 . L_{\text {cut }} H$ is $0.1,1$, 5, 10 from top to bottom [28].

of $M_{c}$ on time. Another moment $\eta_{A}^{\mathrm{C}}$ at which the mode $A$ crosses the horizon is again given by the equation $a\left(\eta_{A}^{\mathrm{C}}\right) / k_{A}=1 / H$. Then the inequality $\eta_{A}^{\mathrm{Q}} \gtrless \eta_{A}^{\mathrm{C}}$ is translated in terms of $M_{c}(\eta)$ as

$$
\eta_{A}^{\mathrm{Q}} \gtrless \eta_{A}^{\mathrm{C}} \Longleftrightarrow \frac{H}{M_{c}\left(\eta_{A}^{\mathrm{Q}}\right)} \gtrless 1
$$

because

$$
\eta_{A}^{\mathrm{Q}} \gtrless \eta_{A}^{\mathrm{C}} \Longleftrightarrow \frac{a\left(\eta_{A}^{\mathrm{Q}}\right)}{k_{A}} \gtrless \frac{a\left(\eta_{A}^{\mathrm{C}}\right)}{k_{A}} \Longleftrightarrow \frac{1}{M_{c}\left(\eta_{A}^{\mathrm{Q}}\right)} \gtrless \frac{1}{H} .
$$

Here we have used the fact that $a(\eta)$ is a monotonically increasing function. Together with the discussion given in subsection 2.2, we thus conclude that the contribution of a mode $A$ to the CMB anisotropy will be suppressed largely if the inequality $H / M_{c}\left(\eta_{A}^{\mathrm{Q}}\right)>1$ holds strongly. Contrarily the contribution of a mode $A$ will be almost the same as in the case with the Bunch-Davies vacuum if the inequality $H / M_{c}\left(\eta_{A}^{\mathrm{Q}}\right)<1$ holds strongly.

How the CMB anisotropy deviates from a scale invariant one depends on the $\eta$ dependence of $M_{c}$. If, for example, $M_{c}(\eta)$ is a monotonically increasing function, then there is a critical time $\eta_{*}$ such that $M_{c}\left(\eta_{*}\right)=H$, and we have the inequality $H / M_{c}(\eta) \gtrless 1$ for $\eta \lessgtr \eta_{*}$. Since larger-scale modes are created at earlier times, the inequality $H / M_{c}\left(\eta_{A}^{\mathrm{Q}}\right)>1$ holds more strongly for larger-scale modes, and thus their contributions to the primordial spectrum are more suppressed, leading to a large-scale damping. On the other hand, if $M_{c}(\eta)$ 
is a monotonically decreasing function, there will be a damping on fluctuations on small scales. If $M_{c}$ is constant and $H / M_{c} \gg 1$ there is no CMB anisotropy, and if $H / M_{c} \ll 1$ we get an ordinary, scale invariant spectrum.

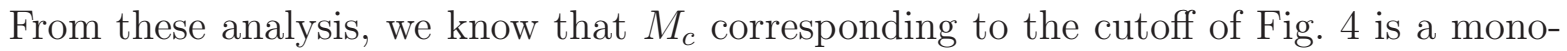
tonically increasing function, and $M_{c}$ corresponding to Fig. 5 is a monotonically decreasing function. $\quad M_{c}$ corresponding to Figs. [6] and [7 are constant, and $H / M_{c}>1, H / M_{c}<1$, respectively.

For example, if we take the homogeneous cutoff used in subsection 2.3.1, then

$$
\eta_{A}^{\mathrm{Q}}=-\frac{1}{k L_{\text {cut }} H}
$$

and thus we have

$$
\frac{1}{M_{c}\left(\eta_{A}^{\mathrm{Q}}\right)}=\frac{a\left(\eta_{A}^{\mathrm{Q}}\right)}{k}=L_{\text {cut }} .
$$

Therefore $M_{c}$ does not depend on $\eta$ and because we make analysis in de Sitter spacetime, we get a scale invariant power spectrum. However, if we take the cutoff used in subsection 2.3.2, then

$$
\eta_{A}^{\mathrm{Q}}=-\frac{r_{*}}{L_{\text {cut }} H} \sqrt{\frac{4 \pi}{l+1}}
$$

and thus we have

$$
M_{c}\left(\eta_{A}^{\mathrm{Q}}\right)=\left(\frac{a\left(\eta_{A}^{\mathrm{Q}}\right)}{k}\right)^{-1}=-H \eta_{A}^{\mathrm{Q}} k \sim-\frac{2 \pi^{2} r_{*}}{L_{\text {cut }}^{2} H \eta_{A}^{\mathrm{Q}}}
$$

where we have used the relation that $k \sim \pi(l+1) / 2 r_{*}$. Therefore $M_{c}(\eta)$ is a monotonically increasing function of $\eta$, giving a large-scale damping.

\section{Analysis without resorting to the classicalization process}

In the preceding section, the large-scale damping is analyzed by comparing the two moments $\eta_{A}^{\mathrm{Q}}$ and $\eta_{A}^{\mathrm{C}}$ for each quantum mode $A$ and also by resorting to the classicalization process of its quantum fluctuation. Although the discussion given there should yield a qualitatively correct result, it is not satisfactory because the classicalization proceeds only gradually around the moment when the mode crosses the horizon, and because the process should be automatically incorporated once the field is quantized with a proper initial condition. 
Furthermore, in the analysis made in subsection 2.3.2, the classical fluctuation of a mode is assumed to take the same magnitude with that in the absence of cutoff once the mode has a period of quantum fluctuation. However, if the mode has only a short period of quantum fluctuation, then only a small magnitude of classical fluctuation will be left after the classicalization process (the fossil should be small if the lifetime of quantum fluctuation is short). Thus in this section, we reanalyze the angular power spectrum of the CMB anisotropy by imposing on each mode an initial condition which seems plausible.

The initial condition we choose is such that each mode $A$ starts its quantum fluctuation as a vacuum fluctuation at the ground state of the Hamiltonian. ${ }^{4}$ We also assume that a cutoff is introduced in such a way that its physical distance scale $L_{\text {cut }}$ is a time-independent constant. We first consider the case in which a homogeneous cutoff is introduced (case of subsection 2.3.1). Then we consider the case in which the two-sphere at $(\eta, r)$ is replaced by the fuzzy sphere $\tilde{S}^{2}$ (case of subsection 2.3.2).

\subsection{Homogeneous cutoff on the physical wave length}

In this subsection, we label the mode as $A=\boldsymbol{k}$ and set the homogeneous cutoff (2.27). We require that at the moment (2.29),$\eta_{A}^{\mathrm{Q}}=-1 / k L_{\text {cut }} H$, the mode $A=\boldsymbol{k}$ be at the ground state of the Hamiltonian.

The action we consider is

$$
S_{\text {fluct }}=\frac{1}{2} \int d \eta d^{3} \boldsymbol{x} \frac{1}{H^{2} \eta^{2}}\left[\left(\partial_{\eta} \phi(\eta, \boldsymbol{x})\right)^{2}-\left(\partial_{i} \phi(\eta, \boldsymbol{x})\right)^{2}\right] .
$$

The conjugate momentum to $\phi$ is $\pi=\partial_{\eta} \phi / H^{2} \eta^{2}$. By introducing the annihilation and creation operators as

$$
\begin{gathered}
\phi=\int \frac{d^{3} \boldsymbol{k}}{(2 \pi)^{3}} \frac{1}{\sqrt{2 \mu_{k}}}\left(a_{\boldsymbol{k}}+a_{-\boldsymbol{k}}^{\dagger}\right) e^{i \boldsymbol{k} \cdot \boldsymbol{x}}, \quad \pi=-i \int \frac{d^{3} \boldsymbol{k}}{(2 \pi)^{3}} \sqrt{\frac{\mu_{k}}{2}}\left(a_{\boldsymbol{k}}-a_{-\boldsymbol{k}}^{\dagger}\right) e^{i \boldsymbol{k} \cdot \boldsymbol{x}}, \\
{\left[a_{\boldsymbol{k}}, a_{\boldsymbol{k}^{\prime}}^{\dagger}\right]=(2 \pi)^{3} \delta^{3}\left(\boldsymbol{k}-\boldsymbol{k}^{\prime}\right),}
\end{gathered}
$$

the Hamiltonian can be written as

$$
\begin{aligned}
H & =\int \frac{d^{3} \boldsymbol{k}}{(2 \pi)^{3}}\left[\omega_{k} a_{\boldsymbol{k}}^{\dagger} a_{\boldsymbol{k}}+\frac{\lambda_{k}}{2}\left(a_{\boldsymbol{k}}^{\dagger} a_{-\boldsymbol{k}}^{\dagger}+a_{\boldsymbol{k}} a_{-\boldsymbol{k}}\right)\right], \\
\omega_{k} & =\frac{\mu_{k}}{2} H^{2} \eta^{2}+\frac{1}{2 \mu_{k}} \frac{k^{2}}{H^{2} \eta^{2}}, \quad \lambda_{k}=-\frac{\mu_{k}}{2} H^{2} \eta^{2}+\frac{1}{2 \mu_{k}} \frac{k^{2}}{H^{2} \eta^{2}} .
\end{aligned}
$$

\footnotetext{
${ }^{4}$ This type of initial condition is investigated in Ref. [18 in a different context.
} 
We choose $\mu_{k}$ such that the Hamiltonian is diagonalized at the moment $\eta_{A}^{\mathrm{Q}}$,

$$
\mu_{k}=\frac{k}{H^{2}\left(\eta_{A}^{Q}\right)^{2}} .
$$

Then our requirement that the mode $\boldsymbol{k}$ be at the ground state when $\eta=\eta_{A}^{\mathrm{Q}}$ can be realized by the following initial condition:

$$
a_{\boldsymbol{k}}\left(\eta_{A}^{\mathrm{Q}}\right)|0\rangle=0
$$

In order to calculate the two-point correlation functions at the exit time of inflation, we take the Heisenberg picture for the annihilation-creation operators and consider their Bogoliubov transformations:

$$
\left(\begin{array}{c}
a_{\boldsymbol{k}}(\eta) \\
a_{-\boldsymbol{k}}^{\dagger}(\eta)
\end{array}\right)=\left(\begin{array}{cc}
A_{\boldsymbol{k}}(\eta) & B_{\boldsymbol{k}}(\eta) \\
B_{-\boldsymbol{k}}^{*}(\eta) & A_{-\boldsymbol{k}}^{*}(\eta)
\end{array}\right)\left(\begin{array}{c}
a_{\boldsymbol{k}}\left(\eta_{A}^{\mathrm{Q}}\right) \\
a_{-\boldsymbol{k}}^{\dagger}\left(\eta_{A}^{\mathrm{Q}}\right)
\end{array}\right) .
$$

The coefficients $A_{k}$ and $B_{-k}^{*}$ are determined by the Heisenberg equations, and using the combinations $\xi_{\boldsymbol{k}} \equiv A_{\boldsymbol{k}}+B_{-\boldsymbol{k}}^{*}$ and $\zeta_{\boldsymbol{k}} \equiv A_{\boldsymbol{k}}-B_{-\boldsymbol{k}}^{*}$, we can express the power spectrum $P_{\phi}(k)$ of the inflaton field at the exit time of inflation $(\eta=-0)$ as

$$
\left\langle 0\left|\phi_{\boldsymbol{k}}^{\dagger}(0) \phi_{\boldsymbol{k}^{\prime}}(0)\right| 0\right\rangle \equiv \frac{2 \pi^{2}}{k^{3}} P_{\phi}(k)(2 \pi)^{3} \delta^{3}\left(\boldsymbol{k}-\boldsymbol{k}^{\prime}\right)=\frac{1}{2 \mu_{k}}\left|\xi_{\boldsymbol{k}}(0)\right|^{2}(2 \pi)^{3} \delta^{3}\left(\boldsymbol{k}-\boldsymbol{k}^{\prime}\right),
$$

or

$$
P_{\phi}(k)=\frac{k^{3}}{(2 \pi)^{2} \mu_{k}}\left|\xi_{\boldsymbol{k}}(0)\right|^{2}
$$

By using the identity,

$$
e^{i \boldsymbol{k x}}=4 \pi \sum_{l m} i^{l} j_{l}(k r) Y_{l m}^{*}\left(\Omega_{\boldsymbol{k}}\right) Y_{l m}(\Omega)
$$

$P_{\phi}(k)$ can be related to the angular power spectrum $C_{l}^{\phi}\left(0, r_{*}\right)$ as

$$
C_{l}^{\phi}\left(0, r_{*}\right)=4 \pi \int_{0}^{\infty} \frac{d k}{k}\left(j_{l}\left(k r_{*}\right)\right)^{2} P_{\phi}(k) .
$$

Note that a scale invariant $P_{\phi}(k)$ leads to $C_{l}^{\phi}\left(0, r_{*}\right) \propto 1 / l(l+1)$. The Heisenberg equations give the following equations :

$$
\ddot{\xi}_{k}(\eta)-\frac{2}{\eta} \dot{\xi}_{\boldsymbol{k}}(\eta)+k^{2} \xi_{\boldsymbol{k}}(\eta)=0, \quad \xi_{\boldsymbol{k}}\left(\eta_{A}^{\mathrm{Q}}\right)=1, \quad \dot{\xi}_{\boldsymbol{k}}\left(\eta_{A}^{\mathrm{Q}}\right)=-i k .
$$


They can be solved with the spherical Hankel function as

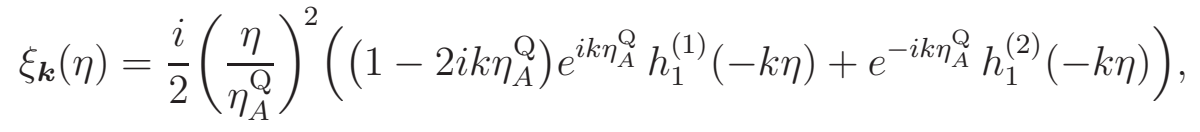

and thus, the power spectrum is determined as

$$
P_{\phi}(k)=\left(\frac{H}{2 \pi}\right)^{2} \frac{1}{4\left(k \eta_{A}^{\mathrm{Q}}\right)^{2}}\left|1-2 i k \eta_{A}^{\mathrm{Q}}-\exp \left(-2 i k \eta_{A}^{\mathrm{Q}}\right)\right|^{2} .
$$

Substituting the equation $\eta_{A}^{\mathrm{Q}}=-1 / k L_{\text {cut }} H$, we get

$$
\begin{aligned}
\left.P_{\phi}(k)\right|_{\eta_{A}^{\mathrm{Q}}=-1 / k L_{\mathrm{cut}} H} & =\left(\frac{H}{2 \pi}\right)^{2} \frac{\left(L_{\mathrm{cut}} H\right)^{2}}{4}\left|1+\frac{2 i}{L_{\mathrm{cut}} H}-\exp \left(\frac{2 i}{L_{\mathrm{cut}} H}\right)\right|^{2} \\
( & =k \text {-independent }) .
\end{aligned}
$$

Thus we obtain a scale invariant power spectrum for the homogeneous cutoff in our approximation that the background metric is a pure de Sitter universe. This result is consistent with a qualitative argument given in subsection 2.3.1. If we take the limits $L_{\text {cut }} H \rightarrow 0$ and $L_{\text {cut }} H \rightarrow \infty$ in Eq. (3.16) we get

$$
\begin{aligned}
\left.P_{\phi}\right|_{L_{\mathrm{cut}} H \rightarrow 0} & =\left(\frac{H}{2 \pi}\right)^{2}, \\
\left.P_{\phi}\right|_{L_{\mathrm{cut}} H \rightarrow \infty} & =0 .
\end{aligned}
$$

This is an expected behavior for a proper initial condition, as was discussed in subsection 2.2 .

\subsection{Cutoff with fuzzy sphere $\tilde{S}^{2}$}

In this subsection, we consider the model considered in subsection 2.3.2, in which threedimensional space is described by $\boldsymbol{R}_{+} \times \tilde{S}^{2}$ (radial coordinate $r$ times a fuzzy sphere). The noncommutativity is introduced only to the angular directions $\Omega$, since this would be most relevant to any modifications to angular power spectrum. ${ }^{5}$ One can also give a holographic interpretation to this way of introducing cutoff, which is briefly commented in conclusion and will be discussed more elaborately in a forthcoming paper [35].

\footnotetext{
${ }^{5}$ In fact, recently it has been shown in Refs. [19, 20] that there is no sharp damping at large angular scales if one introduces noncommutativity only to the time and radial coordinates.
} 


\subsubsection{Coordinates}

As was explained in subsection 2.3.2, the mode with the multipole $l$ starts its quantum fluctuation at the moment (2.35) which depends also on the value of the radial coordinate $r$. Thus, we introduce the new coordinates $(\tau, \sigma, \Omega)$ such that the modes with the same $l$ start at the same time:

$$
H \eta=-e^{\sigma} f_{1}(\tau), \quad H r=e^{\sigma} f_{2}(\tau) .
$$

We here assume that $f_{1}, f_{2}>0$ and that $\partial_{\tau}\left(f_{2} / f_{1}\right)>0$ so that the orientations of $\eta$ and $\tau$ coincide. ${ }^{6}$ Note that

$$
\partial_{\sigma}=\eta \partial_{\eta}+r \partial_{r}
$$

is the Killing vector of the de Sitter metric, so that the metric does not depend on $\sigma$. Setting $p$ to be the conjugate momentum to the coordinate $\sigma$, we label the mode as $A=(p, l, m)$. The cutoff is set on the multipole $l$ as in Eq. (2.33) and we denote by $\tau=\tau_{A}^{\mathrm{Q}}$ the moment when the mode $A=(p, l, m)$ starts to fluctuate quantum mechanically (see Fig. 12):

$$
\left.\frac{f_{2}}{f_{1}}\right|_{\tau=\tau_{A}^{\mathrm{Q}}}=L_{\mathrm{cut}} H \sqrt{\frac{l+1}{4 \pi}} \quad\left(=\alpha_{l}\right) .
$$

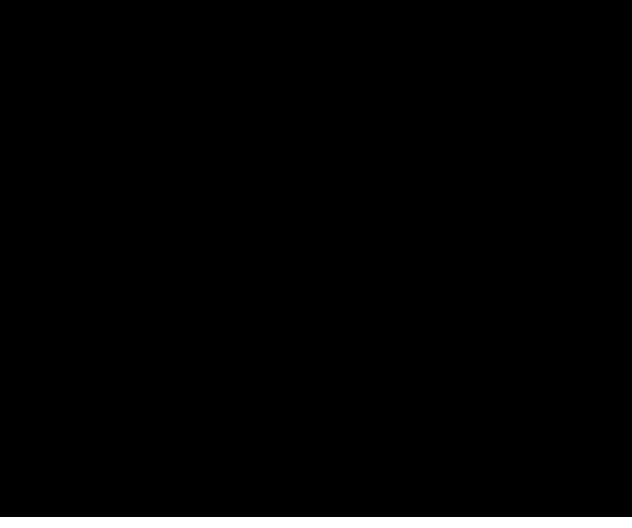

Figure 12: New coordinates $(\tau, \sigma)$. The moment when the mode $A=(p, l, m)$ newly appears is expressed by a single time-slice.

\footnotetext{
${ }^{6}$ Recall that $\eta<0$ during inflation.
} 


\subsubsection{Calculation of the angular power spectrum}

We now calculate the angular power spectrum. We will see that it is independent of the explicit form of the functions $f_{1}$ and $f_{2}$.

Under the above parametrization, the metric is rewritten as

$$
\begin{aligned}
d s^{2} & =\frac{1}{H^{2} \eta^{2}}\left(-d \eta^{2}+d r^{2}+r^{2} d \Omega^{2}\right) \\
& =\frac{1}{H^{2} f_{1}^{2}}\left(\left(\dot{f}_{2}^{2}-\dot{f}_{1}^{2}\right) d \tau^{2}+\left(f_{2}^{2}-f_{1}^{2}\right) d \sigma^{2}+2\left(f_{2} \dot{f}_{2}-f_{1} \dot{f}_{1}\right) d \tau d \sigma+f_{2}^{2} d \Omega^{2}\right) .
\end{aligned}
$$

Here the dot stands for the differentiation with respect to $\tau$. The action can be expanded in the angular modes $(l, m)$. Due to the rotational invariance, it is enough to consider the case $m=0$ :

$$
\begin{aligned}
& S_{\text {fluct }(l, 0)}=\int d \tau d \sigma \frac{f_{2}^{2}}{2 H^{2} f_{1}^{2} F} \times \\
& \times\left[\left(f_{2}^{2}-f_{1}^{2}\right)\left(\partial_{\tau} \phi_{l 0}\right)^{2}+\left(\dot{f}_{2}^{2}-\dot{f}_{1}^{2}\right)\left(\partial_{\sigma} \phi_{l 0}\right)^{2}-2\left(f_{2} \dot{f}_{2}-f_{1} \dot{f}_{1}\right) \partial_{\tau} \phi_{l 0} \partial_{\sigma} \phi_{l 0}-\frac{F^{2} l(l+1)}{f_{2}^{2}} \phi_{l 0}^{2}\right],
\end{aligned}
$$

where $F \equiv f_{1} \dot{f}_{2}-f_{2} \dot{f}_{1}=f_{1}^{2} \partial_{\tau}\left(f_{2} / f_{1}\right)>0$. The conjugate momentum to $\phi_{l 0}$ is

$$
\pi_{l 0}=\frac{f_{2}^{2}}{H^{2} f_{1}^{2} F}\left[\left(f_{2}^{2}-f_{1}^{2}\right) \partial_{\tau} \phi_{l 0}-\left(f_{2} \dot{f}_{2}-f_{1} \dot{f}_{1}\right) \partial_{\sigma} \phi_{l 0}\right]
$$

By introducing the annihilation and creation operators as

$$
\begin{gathered}
\phi_{l 0}=\int_{-\infty}^{\infty} \frac{d p}{2 \pi} \frac{1}{\sqrt{2 \mu_{p}}}\left(a_{p}+a_{-p}^{\dagger}\right) e^{i p \sigma}, \quad \pi_{l 0}=-i \int_{-\infty}^{\infty} \frac{d p}{2 \pi} \sqrt{\frac{\mu_{p}}{2}}\left(a_{p}-a_{-p}^{\dagger}\right) e^{i p \sigma}, \\
{\left[a_{p}, a_{p^{\prime}}^{\dagger}\right]=2 \pi \delta\left(p-p^{\prime}\right),}
\end{gathered}
$$

the Hamiltonian is given by

$$
H=\int_{-\infty}^{\infty} \frac{d p}{2 \pi}\left(\omega_{p} a_{p}^{\dagger} a_{p}+\frac{\lambda_{p}}{2}\left(a_{p}^{\dagger} a_{-p}^{\dagger}+a_{p} a_{-p}\right)\right)
$$

with

$$
\begin{aligned}
\omega_{p} & \equiv \frac{\mu_{p} H^{2} f_{1}^{2} F}{2 f_{2}^{2}\left(f_{2}^{2}-f_{1}^{2}\right)}+\frac{f_{2}^{2} F}{2 \mu_{p} H^{2} f_{1}^{2}}\left(\frac{p^{2}}{f_{2}^{2}-f_{1}^{2}}+\frac{l(l+1)}{f_{2}^{2}}\right)-p \frac{f_{2} \dot{f}_{2}-f_{1} \dot{f}_{1}}{f_{2}^{2}-f_{1}^{2}}, \\
\lambda_{p} & \equiv-\frac{\mu_{p} H^{2} f_{1}^{2} F}{2 f_{2}^{2}\left(f_{2}^{2}-f_{1}^{2}\right)}+\frac{f_{2}^{2} F}{2 \mu_{p} H^{2} f_{1}^{2}}\left(\frac{p^{2}}{f_{2}^{2}-f_{1}^{2}}+\frac{l(l+1)}{f_{2}^{2}}\right) .
\end{aligned}
$$


As in the previous subsection, we set $\mu_{p}$ such that the Hamiltonian is diagonalized at $\tau=\tau_{A}^{\mathrm{Q}}$, making the mode to start its vacuum fluctuation at the ground state:

$$
\begin{aligned}
& \mu_{p}=\left.\frac{f_{2}^{2}}{H^{2} f_{1}^{2}} \sqrt{p^{2}+\frac{f_{2}^{2}-f_{1}^{2}}{f_{2}^{2}} l(l+1)}\right|_{\tau=\tau_{A}^{\mathrm{Q}}}, \\
& a_{p}\left(\tau_{A}^{\mathrm{Q}}\right)|0\rangle=0 .
\end{aligned}
$$

We again consider the Bogoliubov transformations:

$$
\left(\begin{array}{c}
a_{p}(\tau) \\
a_{-p}^{\dagger}(\tau)
\end{array}\right)=\left(\begin{array}{cc}
A_{p}(\tau) & B_{p}(\tau) \\
B_{-p}^{*}(\tau) & A_{-p}^{*}(\tau)
\end{array}\right)\left(\begin{array}{c}
a_{p}\left(\tau_{A}^{\mathrm{Q}}\right) \\
a_{-p}^{\dagger}\left(\tau_{A}^{\mathrm{Q}}\right)
\end{array}\right)
$$

and introduce $\xi_{p} \equiv A_{p}+B_{-p}^{*}$ and $\zeta_{p} \equiv A_{p}-B_{-p}^{*}$, which satisfy the differential equations

$$
\begin{aligned}
& \partial_{\tau} \xi_{p}=-\frac{i}{2}\left(\omega_{p}-\omega_{-p}\right) \xi_{p}-\frac{i}{2}\left(\omega_{p}+\omega_{-p}-2 \lambda_{p}\right) \zeta_{p}, \\
& \partial_{\tau} \zeta_{p}=-\frac{i}{2}\left(\omega_{p}+\omega_{-p}+2 \lambda_{p}\right) \xi_{p}-\frac{i}{2}\left(\omega_{p}-\omega_{-p}\right) \zeta_{p},
\end{aligned}
$$

with the initial conditions

$$
\xi_{p}\left(\tau_{A}^{\mathrm{Q}}\right)=\zeta_{p}\left(\tau_{A}^{\mathrm{Q}}\right)=1
$$

The angular power spectrum is then expressed as

$$
l(l+1) C_{l}^{\phi}=\left.l(l+1)\left\langle 0\left|\phi_{l 0}(\tau, \sigma) \phi_{l 0}(\tau, \sigma)\right| 0\right\rangle\right|_{\eta=-0}=\left.\int_{0}^{\infty} \frac{d p}{2 \pi} \frac{l(l+1)}{\mu_{p}}\left|\xi_{p}(\tau)\right|^{2}\right|_{\eta=-0} .
$$

The time-evolution of $\left|\xi_{p}(\tau)\right|^{2}$ can be evaluated numerically. This can be carried out easily if one notes that the functions

$$
\begin{aligned}
K_{p} & \equiv \frac{l(l+1)}{\mu_{p} H^{2}}\left|\xi_{p}(\tau)\right|^{2} \\
L_{p} & \equiv \frac{i}{2} l(l+1)\left(\xi_{p}^{*}(\tau) \zeta_{p}(\tau)-\xi_{p}(\tau) \zeta_{p}^{*}(\tau)\right) \\
M_{p} & \equiv l(l+1) \mu_{p} H^{2}\left|\zeta_{p}(\tau)\right|^{2}
\end{aligned}
$$

satisfy the following first-order equations:

$$
\begin{aligned}
\partial_{t} K_{p} & =-\frac{2}{t^{2}\left(t^{2}-1\right)} L_{p}, \\
\partial_{t} L_{p} & =\left(\frac{t^{2} p^{2}}{t^{2}-1}+l(l+1)\right) K_{p}-\frac{1}{t^{2}\left(t^{2}-1\right)} M_{p}, \\
\partial_{t} M_{p} & =2\left(\frac{t^{2} p^{2}}{t^{2}-1}+l(l+1)\right) L_{p},
\end{aligned}
$$


with the initial conditions

$$
\begin{gathered}
K_{p}\left(t_{A}^{\mathrm{Q}}\right)=\frac{l(l+1)}{\mu_{p} H^{2}}, \quad L_{p}\left(t_{A}^{\mathrm{Q}}\right)=0, \quad M_{p}\left(t_{A}^{\mathrm{Q}}\right)=l(l+1) \mu_{p} H^{2}, \\
\mu_{p} H^{2}=t_{A}^{\mathrm{Q}} \sqrt{\left(t_{A}^{\mathrm{Q}}\right)^{2} p^{2}+\left(\left(t_{A}^{\mathrm{Q}}\right)^{2}-1\right) l(l+1) .}
\end{gathered}
$$

Here we have introduced a new variable $t$ as

$$
t \equiv-\frac{r}{\eta}=\frac{f_{2}}{f_{1}}
$$

which takes the following value at the initial time:

$$
t_{A}^{\mathrm{Q}} \equiv-\frac{r}{\eta_{A}^{\mathrm{Q}}}=\frac{f_{2}\left(\tau_{A}^{\mathrm{Q}}\right)}{f_{1}\left(\tau_{A}^{\mathrm{Q}}\right)}=L_{\text {cut }} H \sqrt{\frac{l+1}{4 \pi}} .
$$

We also have used the relation

$$
\frac{F}{f_{1}^{2}}=\partial_{\tau} t
$$

The exit time of inflation $(\eta \rightarrow-0)$ corresponds to the limit $t \rightarrow+\infty$. We thus have

$$
l(l+1) C_{l}^{\phi}=\left.H^{2} \int_{0}^{\infty} \frac{d p}{2 \pi} K_{p}(t)\right|_{t=+\infty} .
$$

Since all the expressions (3.40)-(3.44), (3.46) and (3.48) are written in terms of $t$, the angular power spectrum $C_{l}^{\phi}$ is actually independent of the choices of $f_{1}$ and $f_{2}$.

We carried out a numerical calculation and have obtained the angular power spectrum given in Fig. 13, Figure 14 is a magnified figure for small $l$, where the power spectra obtained with the ansatz (2.25) are also drawn for comparison (the spectral index is set to be one for both). The spectra in Fig. 13 are oscillating with $l$ and approach one asymptotically. This oscillation is a common feature of the power spectra when taking initial conditions other than the Bunch-Davies vacuum. ${ }^{7}$ We also see that the resulting power spectra exhibit less sharp damping than the ones obtained with the ansatz (2.25). Although the damping we get here may not be sufficiently large to fit the observational data, the curve could be better fit to the data by taking account of corrections in the slow roll approximation (i.e., including a nontrivial spectral index), or it is still possible that our initial condition needs to be modified. ${ }^{8}$

\footnotetext{
${ }^{7}$ See Refs. 16, 22, 23]. Note that we had no such oscillation in subsection 3.1. It is because we there analyzed the power spectrum with the use of the homogeneous cutoff in de Sitter spacetime.

${ }^{8}$ Of course, another way to understand the result is to consider the cosmic variance (i.e., to regard the observational data as a deviation from the obtained result above).
} 


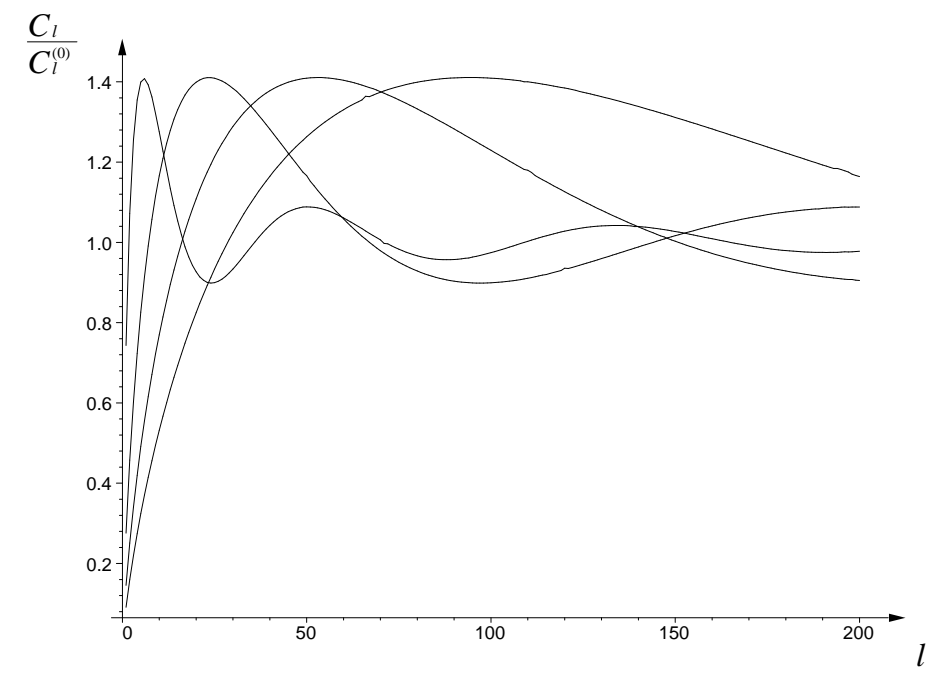

Figure 13: Damping factor for the space $R_{+} \times \tilde{S}^{2}$. The horizontal axis represents the multipole $l$, while the vertical axis is $C_{l} / C_{l}^{(0)}=2 \pi l(l+1) C_{l}^{\phi} / H^{2} . C_{l}^{(0)}$ represents the angular power spectrum in the absence of noncommutativity. Here $L_{\text {cut }} H=5,10,15,20$ from top to bottom. All curves obey the approximate scaling law explained in the main text, and converges to one for large $l$.

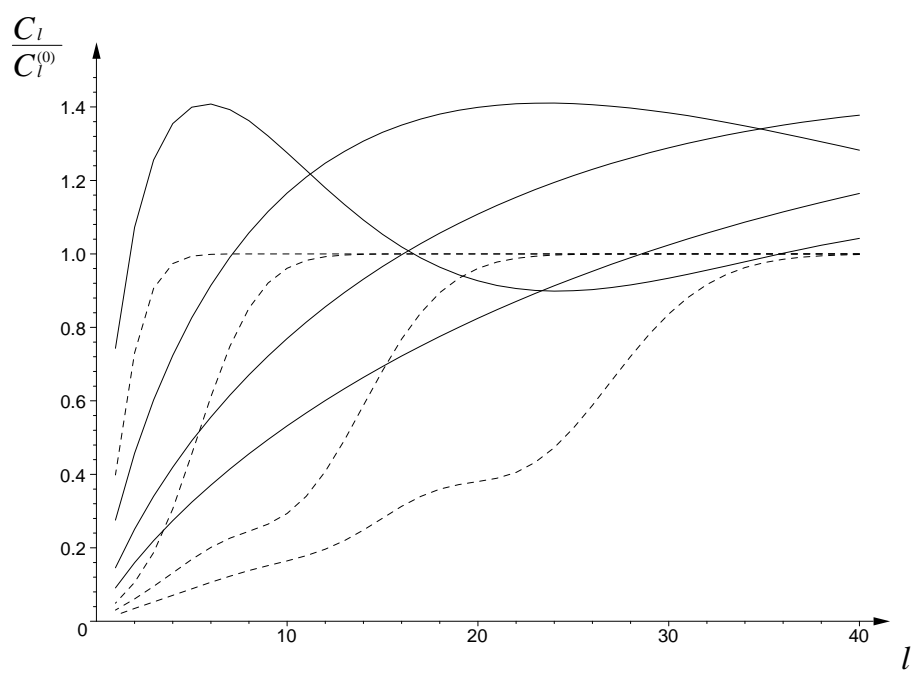

Figure 14: Damping factor for the space $R_{+} \times \tilde{S}^{2}$. The horizontal axis represents the multipole $l$, while the vertical axis is $C_{l} / C_{l}^{(0)}$. Dashed lines are the spectra obtained with the ansatz [2.25), while solid lines are the spectra given in this section. In each set $L_{\text {cut }} H=5,10,15,20$ from top to bottom. Here the spectral index is set to be $n=1$ for both. 


\subsubsection{Scaling law in the angular power spectrum}

As can be seen from Fig. 13, the curves of the angular power spectrum shift to right for larger $L_{\text {cut }} H$. In fact, one can prove that the angular power spectrum is a function of the scaling variable $l /\left(L_{\text {cut }} H\right)^{2}$ with good precision. In order to show this, we assume that $t_{A}^{\mathrm{Q}} \gg 1$ in Eqs. (3.40)-(3.44), ${ }^{9}$ and rescale $p$ and $t$ in Eq. (3.48) such that

$$
p=k \sqrt{l(l+1)}, \quad t=s t_{A}^{\mathrm{Q}} \quad\left(t=t_{A}^{\mathrm{Q}} \Leftrightarrow s=1\right) .
$$

Then by setting

$$
\mathcal{K}_{k} \equiv \sqrt{l(l+1)} K_{p}, \quad \mathcal{L}_{k} \equiv \frac{\sqrt{l(l+1)} L_{p}}{\left(t_{A}^{\mathrm{Q}}\right)^{3}}, \quad \mathcal{M}_{k} \equiv \frac{\sqrt{l(l+1)} M_{p}}{\left(t_{A}^{\mathrm{Q}}\right)^{6}}
$$

all the equations depend only on the combination $l /\left(L_{\text {cut }} H\right)^{2}=l(l+1) / 4 \pi\left(t_{A}^{\mathrm{Q}}\right)^{2}$ and on $k$ :

$$
\begin{aligned}
\partial_{s} \mathcal{K}_{k} & =-\frac{2}{s^{4}} \mathcal{L}_{k} \\
\partial_{s} \mathcal{L}_{k} & =\left(k^{2}+1\right) \frac{l(l+1)}{\left(t_{A}^{\mathrm{Q}}\right)^{2}} \mathcal{K}_{k}-\frac{1}{s^{4}} \mathcal{M}_{k}, \\
\partial_{s} \mathcal{M}_{k} & =2\left(k^{2}+1\right) \frac{l(l+1)}{\left(t_{A}^{\mathrm{Q}}\right)^{2}} \mathcal{L}_{k}
\end{aligned}
$$

with

$$
\mathcal{K}_{k}(1)=\frac{l(l+1)}{\left(t_{A}^{\mathrm{Q}}\right)^{2} \sqrt{\left(k^{2}+1\right)}}, \quad \mathcal{L}_{k}(1)=0, \quad \mathcal{M}_{k}(1)=\sqrt{k^{2}+1} \frac{(l(l+1))^{2}}{\left(t_{A}^{\mathrm{Q}}\right)^{4}} .
$$

Since the angular power spectrum is expressed as the integration of $\mathcal{K}_{k}$ over $k$ :

$$
l(l+1) C_{l}^{\phi}=\left.H^{2} \int_{0}^{\infty} \frac{d p}{2 \pi} K_{p}(t)\right|_{t=+\infty}=\left.H^{2} \int_{0}^{\infty} \frac{d k}{2 \pi} \mathcal{K}_{k}(s)\right|_{s=+\infty},
$$

we conclude that the angular power spectrum is a function only of $l /\left(L_{\text {cut }} H\right)^{2}$ when one can assume that $t_{A}^{\mathrm{Q}} \gg 1$.

\section{Conclusion and outlook}

In this article, we have investigated the effects of cutoff or noncommutativity on the CMB anisotropy, focusing on the possibility that the large-scale damping observed in the CMB angular power spectrum may be explained as such effect.

\footnotetext{
${ }^{9}$ As can be seen from Eq. (3.46), this assumption holds well when $L_{\text {cut }} H$ and/or $l$ are large.
} 
We gave an analysis in two ways: the first was based on the classicalization process, and the second was carried out by analyzing the time-evolution of the Heisenberg operators with the initial condition such that modes start as vacuum fluctuations.

In the first analysis (section 2), we have clarified the mechanism that a short-distance cutoff can affect the CMB anisotropies on large scales. We there introduced two typical moments during inflation, one is the moment $\eta_{A}^{\mathrm{C}}$ when a given mode $A$ crosses the Hubble horizon and becomes classical, and the other is the moment $\eta_{A}^{\mathrm{Q}}$ at which the mode $A$ starts to fluctuate quantum mechanically. We have shown that there exists a large-scale damping if $\eta_{A}^{\mathrm{C}}$ is prior to $\eta_{A}^{\mathrm{Q}}$ only for larger-scale modes. Furthermore, we have demonstrated that the homogeneous three-dimensional cutoff does not yield such damping, while the assumption that space is the product of the radial coordinate and the fuzzy sphere with short-distance cutoff $L_{\text {cut }}$ certainly exhibits the large-scale damping if one assumes that the noncommutative scale is around the Hubble parameter during inflation. ${ }^{10}$

In the second analysis based on the Heisenberg equations (section 3), we again have shown that the homogeneous three-dimensional cutoff only gives rise to the scale invariant power spectrum, while the cutoff based on the fuzzy sphere certainly yields the power spectrum which exhibits the large-scale damping.

We thus may conclude that our analysis presented in this article gives a qualitatively correct explanation on the mechanism of the large-scale damping. However, the figure does not exhibit enough damping compared to the observational data. This would be improved if we introduce a nontrivial spectral index. Another possible way around is to modify our initial condition. In fact, one would demand that when a mode newly appears its amplitude should be as small as possible, but the vacuum fluctuation may not be the least amount of fluctuation among those that are allowed. It would be nice if one could find a proper initial condition which correctly describes the creation of modes after being released from the constraint of cutoff. The initial conditions in chaotic inflation [7] or in the models of "the universe from nothing" [37] would be interesting to investigate in this context. ${ }^{11}$

Another point which should be clarified in the future is the fact that the homogeneous

\footnotetext{
${ }^{10}$ In Ref. [36], a cyclic model of the universe is considered, giving the scenario that there is an era during which the universe expands exponentially with the Hubble parameter equal to the string mass scale.

${ }^{11}$ In searching for desirable initial conditions, one will need to carefully check whether the choice of initial condition avoids the backreaction problem (see for example Refs. 24 25]). In fact, some initial conditions can cause particle creation, and the energy density of the particles could become comparable to the background energy density which was first assumed to be the main source driving inflation.
} 
three-dimensional cutoff does not lead to a large-scale damping while the cutoff based on fuzzy sphere actually does. This may not be satisfactory from the viewpoint of the standard cosmology in the sense that the latter way of cutoff does not respect the cosmic principle or the invariance under the three-dimensional Euclidean group $(E(3)=I S O(3))$. However, from the holographic viewpoint [38, this way of cutoff with the fuzzy sphere would be consistent with the following scenario of inflation. First, since the noncommutative scale in our model is close to the Hubble parameter during inflation, matter fields are strongly coupled to gravitation, so that the holographic nature would dominate the dynamics of the system. We further assume that the holographic principle holds for arbitrary threedimensional spacelike region (not only for lightsheets [39]) if one considers a combined system of matter fields and gravity. Then the dynamical degrees of freedom in any three-dimensional region should be controlled by the information on the boundary. If the inflaton (or any other scalar-like collective mode in the system) represents such degrees of freedom, then the holographic cutoff for any three-dimensional ball would be to set a noncommutativity on the spherical boundary. This line of investigation is now in progress and will be reported elsewhere.

\section{Acknowledgments}

The authors would like to thank P. Freund, T. Kobayashi and S.-H. Tye for useful discussions. This work is supported in part by Grant-in-Aid (No. 15540269) from the Japan Ministry of Education, Culture, Sports, Science and Technology, and by Grant-in-Aid for the 21st Century COE "Center for Diversity and Universality in Physics."

\section{References}

[1] J. R. Bond, A. H. Jaffe and L. Knox, Phys. Rev. D 57 (1998) 2117 arXiv:astro-ph/9708203.

[2] C. L. Bennett et al., arXiv:astro-ph/0302207.

[3] D. N. Spergel et al., arXiv:astro-ph/0302209.

[4] H. V. Peiris et al., arXiv:astro-ph/0302225. 
[5] A. H. Guth, Phys. Rev. D 23 (1981) 347; K. Sato, Phys. Lett. B 99 (1981) 66; Mon. Not. R. Astron. Soc. 195 (1981) 467.

[6] A. D. Linde, Phys. Lett. B 108 (1982) 389; Phys. Lett. B 114 (1982) 431; A. Albrecht and P. J. Steinhardt, Phys. Rev. Lett. 48 (1982) 1220.

[7] A. D. Linde, Phys. Lett. B 129 (1983) 177; Mod. Phys. Lett. A 1 (1986) 81; Phys. Lett. B 175 (1986) 395; A. D. Linde, D. A. Linde and A. Mezhlumian, Phys. Rev. D 49 (1994) 1783 arXiv:gr-qc/9306035.

[8] A. D. Linde, Phys. Lett. B 249 (1990) 18; Phys. Lett. B 259 (1991) 38.

[9] A. Shafieloo and T. Souradeep, arXiv:astro-ph/0312174

[10] G. Efstathiou, Mon. Not. Roy. Astron. Soc. 346 (2003) L26 arXiv:astro-ph/0306431.

[11] G. Efstathiou, arXiv:astro-ph/0310207

[12] A. R. Liddle and D. H. Lyth, "Cosmological Inflation and Large-Scale Structure," Cambridge University Press (2000).

[13] J. Yokoyama, Phys. Rev. D 59 (1999) 107303.

[14] C. R. Contaldi, M. Peloso, L. Kofman and A. Linde, JCAP 0307 (2003) 002 arXiv:astro-ph/0303636.

[15] M. Kawasaki and F. Takahashi, arXiv:hep-ph/0305319.

[16] J. Martin and R. H. Brandenberger, Phys. Rev. D 63 (2001) 123501 arXiv:hep-th/0005209; R. H. Brandenberger and J. Martin, Mod. Phys. Lett. A 16 (2001) 999 arXiv:astro-ph/0005432.

[17] J. C. Niemeyer, Phys. Rev. D 63 (2001) 123502 arXiv:astro-ph/0005533.

[18] R. Brandenberger and P. M. Ho, Phys. Rev. D 66 (2002) 023517 [AAPPS Bull. 12N1 (2002) 10] arXiv:hep-th/0203119.

[19] S. Tsujikawa, R. Maartens and R. Brandenberger, Phys. Lett. B 574 (2003) 141 arXiv:astro-ph/0308169.

[20] Q. G. Huang and M. Li, JCAP 0311 (2003) 001 arXiv:astro-ph/0308458. 
[21] C. S. Chu, B. R. Greene and G. Shiu, Mod. Phys. Lett. A 16 (2001) 2231 arXiv:hep-th/0011241; F. Lizzi, G. Mangano, G. Miele and M. Peloso, JHEP 0206 (2002) 049 arXiv:hep-th/0203099; Q. G. Huang and M. Li, JHEP 0306 (2003) 014 arXiv:hep-th/0304203; Q. G. Huang and M. Li, arXiv:astro-ph/0311378.

[22] S. Cremonini, Phys. Rev. D 68 (2003) 063514 arXiv:hep-th/0305244.

[23] R. Easther, B. R. Greene, W. H. Kinney and G. Shiu, Phys. Rev. D 67 (2003) 063508 arXiv:hep-th/0110226; S. Shankaranarayanan, Class. Quant. Grav. 20 (2003) 75 arXiv:gr-qc/0203060; U. H. Danielsson, Phys. Rev. D 66 (2002) 023511 arXiv:hep-th/0203198; R. Easther, B. R. Greene, W. H. Kinney and G. Shiu, Phys. Rev. D 66 (2002) 023518 arXiv:hep-th/0204129; L. Bergstrom and U. H. Danielsson, JHEP 0212 (2002) 038 arXiv:hep-th/0211006; G. L. Alberghi, R. Casadio and A. Tronconi, Phys. Lett. B 579 (2004) 1 arXiv:gr-qc/0303035; J. Martin and R. Brandenberger, Phys. Rev. D 68 (2003) 063513 arXiv:hep-th/0305161; O. Elgaroy and S. Hannestad, Phys. Rev. D 68 (2003) 123513 arXiv:astro-ph/0307011; J. Martin and C. Ringeval, arXiv:astro-ph/0310382.

[24] T. Tanaka, arXiv:astro-ph/0012431.

[25] N. Kaloper, M. Kleban, A. Lawrence, S. Shenker and L. Susskind, JHEP 0211 (2002) 037 arXiv:hep-th/0209231.

[26] G. Veneziano, Europhys. Lett. 2 (1986) 199; D. J. Gross and P. F. Mende, Nucl. Phys. B 303 (1988) 407; D. Amati, M. Ciafaloni and G. Veneziano, Phys. Lett. B 216 (1989) 41; R. Guida, K. Konishi and P. Provero, Mod. Phys. Lett. A 6 (1991) 1487.

[27] T. Yoneya, in "Wandering in the Fields", eds. K. Kawarabayashi, A. Ukawa (World Scientific, 1987 ), p.419; M. Li and T. Yoneya, Phys. Rev. Lett. 78 (1997) 1219 arXiv:hep-th/9611072; T. Yoneya, Prog. Theor. Phys. 103 (2000) 1081 arXiv:hep-th/0004074.

[28] M. Fukuma, Y. Kono and A. Miwa, Nucl. Phys. B 682 (2004) 377 arXiv:hep-th/0307029.

[29] N. D. Birrell and P. C. W. Davies, "Quantum Fields in Curved Space," Cambridge University Press (1982); P. K. Townsend, arXiv:gr-qc/9707012.

[30] T. M. Davis and C. H. Lineweaver, arXiv:astro-ph/0310808. 
[31] A. Albrecht, P. Ferreira, M. Joyce and T. Prokopec, Phys. Rev. D 50 (1994) 4807 arXiv:astro-ph/9303001.

[32] R. Sachs and A. Wolfe, Astrophys. J. 147 (1967) 73.

[33] J. M. Bardeen, Phys. Rev. D 22 (1980) 1882; H. Kodama and M. Sasaki, Prog. Theor. Phys. Suppl. 78 (1984) 1; V. F. Mukhanov, H. A. Feldman and R. H. Brandenberger, Phys. Rept. 215 (1992) 203.

[34] J. Hoppe, PhD Thesis (1982), Soryushiron Kenkyu (Kyoto) 80 (1989) 145; J. Hoppe and H. Nicolai, Phys. Lett. B 196 (1987) 451; B. de Wit, J. Hoppe and H. Nicolai, Nucl. Phys. B 305 (1988) 545; J. Madore, Class. Quantum Grav. 9 (1992) 69.

[35] M. Fukuma, Y. Kono and A. Miwa, work in progress.

[36] M. Fukuma, H. Kawai and M. Ninomiya, to appear in Int. J. Mod. Phys. A arXiv:hep-th/0307061.

[37] A. Vilenkin, Phys. Lett. B 117 (1982) 25.

[38] G. 't Hooft, arXiv:gr-qc/9310026 L. Susskind, J. Math. Phys. 36 (1995) 6377 arXiv:hep-th/9409089; R. Bousso, Rev. Mod. Phys. 74 (2002) 825 arXiv:hep-th/0203101.

[39] R. Bousso, JHEP 9907 (1999) 004 arXiv:hep-th/9905177); R. Bousso, JHEP 9906 (1999) 028 arXiv:hep-th/9906022. 